\title{
Descripción clínica y tratamiento de los pacientes con síndrome coronario agudo
}

\section{Clinical description and treatment of patients with acute coronary syndrome}

\author{
John Jaime Sprockel, Juan José Diaztagle, Viviana Carolina Filizzola, \\ Laura Patricia Uribe, Camilo Andrés Alfonso • Bogotá, D.C. (Colombia)
}

\section{Resumen}

Antecedentes: el síndrome coronario agudo es una emergencia clínica que se manifiesta principalmente por dolor torácico. Su diagnóstico representa un desafío clínico y su reconocimiento temprano es fundamental para el tratamiento.

Objetivo: describir las características clínicas, identificar abordajes terapéuticos y conocer los desenlaces de los pacientes con diagnóstico definitivo de síndrome coronario agudo hospitalizados por el servicio de medicina interna y cardiología entre el $1^{\circ}$ de enero de 2009 hasta 31 de diciembre de 2010 en el Hospital San José.

Métodos: se realizó una descripción de una cohorte de pacientes con diagnóstico de síndrome coronario agudo (infarto miocárdico con y sin elevación del segmento ST y angina inestable). Se analizaron variables demográficas, características clínicas, tratamiento y desenlaces a los cuales se calcularon promedio, desviación estándar y proporciones según la naturaleza de las mismas.

Resultados: se reportan 133 pacientes. El 63\% correspondió a hombres, el promedio de edad fue de 64.8 años. La frecuencia de eventos fue 45.9\% IMEST, 39\% IMSEST y 15.1\% anginas inestables. El $96.2 \%$ de los pacientes consultó por dolor torácico, $50.7 \%$ presentaron dolor en las primeras seis horas de evolución. El 93\% recibió betabloqueador, 88\% IECA, 87\% clopidogrel y 97\% ASA. Se trombolizó al $82 \%$ de los pacientes en ventana. La mortalidad intrahospitalaria fue del $8.7 \%$.

Conclusiones: en la población estudiada predominó el síndrome coronario agudo sin elevación del segmento ST. La mayoría de pacientes consultaron por dolor torácico retroesternal, en las primeras horas de inicio. El porcentaje de uso de los medicamentos fue adecuado. La reperfusión inmediata en pacientes con IMEST fue alta, sin embargo aún tenemos pacientes que no se benefician de esta terapia y el uso de angioplastias primarias fue muy bajo. (Acta Med Colomb 2014; 39: 124-130).

Palabras clave: dolor torácico, angina, infarto agudo de miocardio, diagnóstico, tratamiento.

\footnotetext{
Abstract

Background: acute coronary syndrome is a medical emergency that is manifested mainly by chest pain. The diagnosis is a clinical challenge and its early recognition is essential for treatment.

Objective: to describe the clinical characteristics, identify therapeutic approaches and recognize the outcomes of patients with a final diagnosis of acute coronary syndrome hospitalized in Internal Medicine and Cardiology services between January 1, 2009 until December 31, 2010 at the Hospital San José.

Methods: description of a cohort of patients diagnosed with acute coronary syndrome (myocardial infarction with and without ST segment elevation and unstable angina) was performed. Demographic variables, clinical characteristics, treatment and outcomes were analyzed and average, standard deviation and proportions were calculated according to their the nature.

Results: 133 patients were reported. $63 \%$ were men, the mean age was 64.8 years. The event rate was $45.9 \%$ STEMI, 39\% NSTEMI and unstable angina $15.1 \% .96 .2 \%$ of patients consulted for chest pain, $50.7 \%$ had pain in the first six hours of evolution. $93 \%$ received beta-blockers, $88 \%$ ACE inhibitors, $87 \%$ clopidogrel and $97 \%$ ASA. $82 \%$ of patients in window underwent thrombolysis. The hospital mortality was $8.7 \%$.
}

Dr. John Jaime Sprockel Díaz: Internista. Instructor Asistente Departamento Medicina Interna, Fundación Universitaria de Ciencias de la Salud, Hospital San José. Candidato a Maestría en Ingeniería de Sistemas y Computación, Pontificia Universidad Javeriana; Dr. Juan José Diaztagle Fernández: Médico Internista, Epidemiólogo, Magíster en Fisiología. Instructor Asistente Departamento de Medicina Interna Fundación Universitaria de Ciencias de la Salud, Hospital San José. Profesor Asociado Departamento de Ciencias Fisiológicas, Universidad Nacional de Colombia; Dra. Viviana Carolina Filizzola Montero: Residente segundo año Medicina Interna Fundación Universitaria de Ciencias de la Salud, Hospital San José; Dra. Laura Patricia Uribe Posso y Dr. Camilo Andrés Alfonso Cortés: Residentes tercer año Medicina Interna. Fundación Universitaria de Ciencias de la Salud, Hospital San José. Departamento Medicina Interna, División de Investigaciones. Fundación Universitaria de Ciencias de la Salud, Hospital San José de Bogotá. Bogotá, D.C. (Colombia). Correspondencia. Dr. John Jaime Sprockel Díaz. Bogotá, D.C. (Colombia). E-mail: jjsprockel@fucsalud.edu.co Recibido: 30/V/2013 Aceptado: 03/III/2014 
Conclusions: in the studied population acute coronary syndrome without ST segment elevation predominated. Most patients consulted by retrosternal chest pain in the early hours of onset. The percentage of drug use was appropriate. Immediate reperfusion in STEMI patients was high; however we still have patients who do not benefit from this therapy and the use of primary angioplasty was very low. (Acta Med Colomb 2014; 39: 124-130).

Keywords: chest pain, angina, myocardial infarction, diagnosis, treatment.

\section{Introducción}

Según datos reportados por la Organización Mundial de la Salud (OMS), durante 2008, la enfermedad coronaria fue la principal causa de muerte no comunicable en el mundo entero, con 17 millones (1). En Colombia, de acuerdo con los reportes del Departamento Administrativo Nacional de Estadística (DANE) en Colombia también constituyeron la principal causa de muerte en pacientes mayores de 65 años durante 2009 y 2010 , ocasionando 27618 y 29394 defunciones respectivamente (2). Estos datos resaltan la importancia que tiene esta patología en la población general.

Una de las formas de presentación de la enfermedad coronaria es el síndrome coronario agudo (SCA), el cual es una emergencia médica que incluye tres entidades con una alta tasa de morbimortalidad: angina inestable, infarto agudo de miocardio sin elevación del ST (IMSEST) e infarto agudo de miocardio con elevación del ST (IMEST). La principal manifestación clínica de este síndrome es el dolor torácico, que es uno de los motivos de consulta más frecuente en el servicio de urgencias y hace parte de las manifestaciones clínicas de otras patologías. Identificar de forma rápida los pacientes con SCA y realizar un tratamiento farmacológico e intervencionista temprano es fundamental para obtener buenos resultados clínicos con disminución de la morbimortalidad (3-5). De hecho, se ha establecido como una prioridad la disminución del tiempo de atención prehospitalaria y en los servicios de urgencias (3).

El conocimiento de los exámenes diagnósticos practicados y el tratamiento recibido en este grupo de pacientes es importante para evaluar el manejo hospitalario como un proceso integral y poder identificar cuáles son los aspectos en los cuales se puede mejorar la calidad de la atención de los mismos. A nivel mundial este es uno de los objetivos de los diversos registros que se han realizado sobre $\operatorname{SCA}(6,7)$.

Adicionalmente, se sabe que el enfoque diagnóstico inicial de estos pacientes es un verdadero desafío para el clínico y a menudo no se realiza un diagnóstico de ingreso adecuado (8). Por lo tanto, es importante conocer las características clínicas de estos pacientes, ya que la historia clínica sigue siendo la base del enfoque diagnóstico en esta patología.

El objetivo de esta investigación es describir las manifestaciones clínicas, las pruebas diagnósticas y el manejo instaurado tanto farmacológico como intervencionista, por parte del servicio de medicina interna y cardiología del Hospital San José de Bogotá en pacientes cuyo diagnóstico de egreso fue algunas de las patologías descritas como SCA.

\section{Material y métodos}

se realizó un estudio descriptivo de una cohorte de pacientes con diagnóstico de SCA, atendidos por el servicio de medicina interna y cardiología del Hospital San José de Bogotá entre el primero de enero de 2009 y el treinta y uno de diciembre de 2010. Se revisaron las historias clínicas de los pacientes del archivo institucional y extrainstitucional con los datos suministrados por el departamento de estadística del hospital, codificadas según diagnóstico CIE 10 (I209, I210, I212, I213, I214, I219). Se incluyeron pacientes mayores de 18 años, admitidos al servicio de urgencias con diagnóstico de egreso de IMEST, IMSEST o angina inestable. Se excluyeron pacientes con diagnósticos finales diferentes no relacionados, o pacientes que ingresaron directamente al servicio de hemodinamia para realización de cateterismo programado.

Se obtuvo información sobre el sexo, edad, presentación clínica del dolor torácico, antecedentes personales (hipertensión arterial, diabetes mellitus tipo 2, dislipidemia, uso de asa, enfermedad coronaria), hallazgos al examen físico (frecuencia cardiaca, presión arterial, auscultación cardiopulmonar), manejo instaurado (oxígeno, inhibidores de la enzima convertidora de angiotensina, ASA, clopidogrel, morfina, nitratos, heparinas, betabloqueadores), intervenciones realizadas (trombolísis, angioplastia), ubicación y estancia hospitalaria de los pacientes, diagnóstico y condición de egreso (vivo o muerto).

La información se anotó en un formato de recolección estructurado para tal fin. Fueron realizadas auditorías al 10\% de los formatos para comprobar la calidad de los datos. Para el análisis de la información se construyó una base de datos en Excel 2007. El análisis estadístico se realizó en STATA 10. Las variables categóricas se expresan con frecuencias absolutas y relativas, las cuantitativas con promedios y desviación estándar. El estudio fue aprobado por el comité de investigaciones y el comité de ética en investigación con seres humanos de la Fundación Universitaria de Ciencias de la Salud y el Hospital San José de Bogotá.

\section{Resultados}

Se identificaron 183 pacientes con diagnóstico de ingreso o egreso de IMEST, IMSEST o angina inestable, de 
los cuales 50 fueron excluidos. Las causas de exclusión se describen en la Figura 1. De esta forma se incluyeron al estudio 133 pacientes, $45.8 \%(\mathrm{n}=61)$ tuvieron diagnóstico de IMEST, 39.1\% $(n=52)$ IMSEST y $15 \%(n=20)$ angina inestable. En la población estudiada, $63.1 \%(n=84)$ fueron hombres, el promedio de edad fue de 64.8 años (DE 13.7 años). Se encontró que $63.2 \%(\mathrm{n}=84)$ tuvieron antecedente de hipertensión arterial, $49.6 \%(\mathrm{n}=66)$ tabaquismo, $15.1 \%$ $(n=20)$ diabetes y $28.5 \%(n=38)$ enfermedad coronaria (ver Tabla 1).

\section{Presentación clínica}

El 96.2\% ( $n=128)$ de los pacientes refirieron dolor torácico el cual se presentó predominantemente de forma opresiva $79 \%(\mathrm{n}=105)$, la mayoría de los pacientes presentó un episodio de dolor torácico en las 24 horas previas al ingreso $86.1 \%(\mathrm{n}=112)$. El dolor se acompañó en $54.1 \%(\mathrm{n}=72)$ de diaforesis y de disnea en $51.8 \%(n=69)$. Teniendo en cuenta que un paciente podía tener más de un sitio de localización del dolor, el dolor retroesternal predominó en $81.2 \%$ $(\mathrm{n}=108)$, seguido por tórax anterior 9.8\% $(\mathrm{n}=13)$ (Tabla 1).

Un 50.7\% ( $\mathrm{n}=64)$ de los pacientes consultó a la institución con menos de seis horas de duración de inicio del dolor torácico. Entre los pacientes con diagnóstico de IMEST54\% $(n=33)$ consultaron en las primeras seis horas de inicio del dolor, $27 \%(n=17)$ lo hicieron en las primeras dos horas (Tabla 1). Al ingreso, se encontró hipotensión en 5.3\% (n=7), bradicardia en $21.1 \%(\mathrm{n}=28)$ y desaturación $\left(\mathrm{SaO}_{2}\right.$ menor

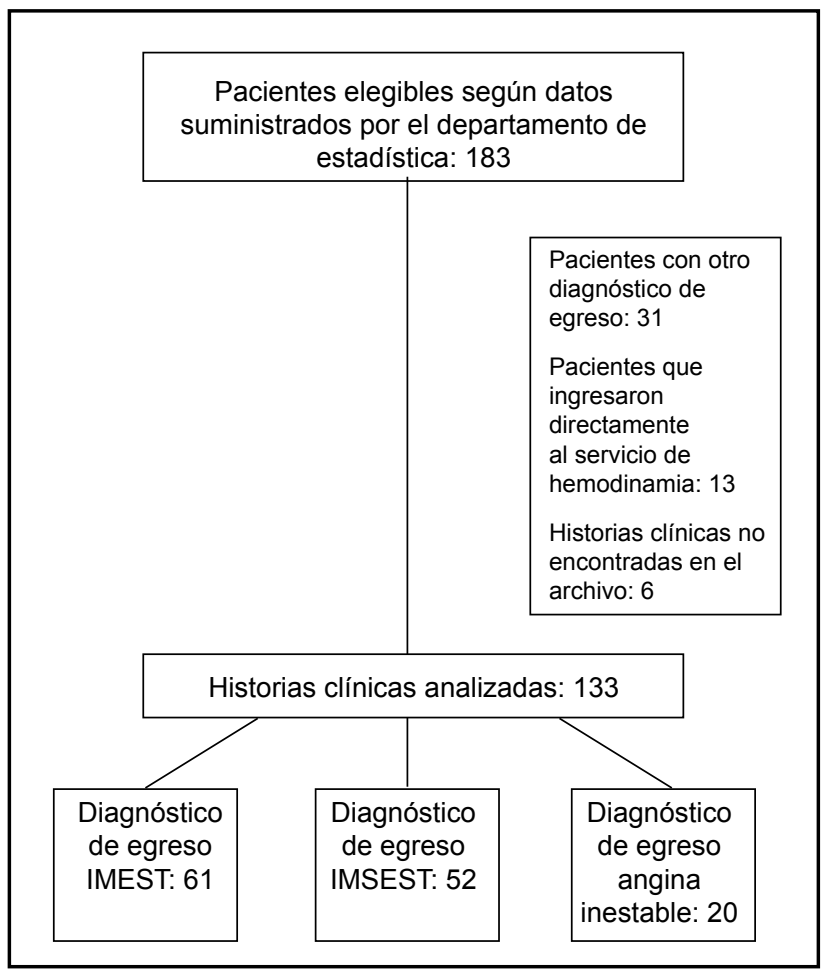

Figura 1. Distribución de los pacientes.
Tabla 1. Características demográficas y clínicas de pacientes con síndrome coronario agudo.

\begin{tabular}{|c|c|c|c|}
\hline & $\begin{array}{c}\text { Total } \\
133\end{array}$ & $\begin{array}{c}\text { IMEST } \\
61\end{array}$ & $\begin{array}{c}\text { SCASEST } \\
72\end{array}$ \\
\hline Edad, promedio (DE) & $64.8(13.7)$ & $62.1(13.6)$ & $67.1(13.3)$ \\
\hline Sexo masculino, $\mathrm{n}(\%)$ & $84(63.1)$ & $47(77)$ & $37(51.4)$ \\
\hline Presencia de dolor, n (\%) & $129(96.9)$ & $61(100)$ & $68(94.4)$ \\
\hline $\begin{array}{l}\text { Características del dolor,*n }(\%) \\
\text { Opresivo } \\
\text { Urente } \\
\text { Punzada } \\
\text { Otro }\end{array}$ & $\begin{array}{l}105(79) \\
16(12) \\
7(5.3) \\
2(1.5)\end{array}$ & $\begin{array}{c}54(88.5) \\
7(11.5) \\
1(1.6) \\
0\end{array}$ & $\begin{array}{l}51(70.8) \\
9(12.5) \\
6(8.3) \\
2(2.7)\end{array}$ \\
\hline $\begin{array}{l}\text { Episodios de dolor torácico en } \\
\text { últimas } 24 \text { horas, } \mathrm{n}(\%) \\
1 \\
2 \\
3 \\
4\end{array}$ & $\begin{array}{c}112(86.1) \\
13(10) \\
4(3.1) \\
1(0.7)\end{array}$ & $\begin{array}{c}50(82) \\
8(13.1) \\
3(4.9) \\
0\end{array}$ & $\begin{array}{c}62(86.1) \\
5(7) \\
1(1.4) \\
1(1.4)\end{array}$ \\
\hline $\begin{array}{l}\text { Duración del dolor, } \mathrm{n}(\%) \\
<6 \mathrm{~h} \\
6-12 \mathrm{~h} \\
>12 \mathrm{~h}\end{array}$ & $\begin{array}{l}64(50.7) \\
17(13.4) \\
45(35.7)\end{array}$ & $\begin{array}{l}33(54) \\
7(11.5) \\
14(23)\end{array}$ & $\begin{array}{l}25(34.7) \\
10(13.8) \\
31(43)\end{array}$ \\
\hline $\begin{array}{l}\text { Asociación del dolor torácico } \dagger \\
\text { Disnea } \\
\text { Diaforesis } \\
\text { Náuseas } \\
\text { Síncope }\end{array}$ & $\begin{array}{l}102(76) \\
69(51.8) \\
72(54.1) \\
53(39.8) \\
7(5.2)\end{array}$ & $\begin{array}{l}52(85.2) \\
35(57.4) \\
41(67.2) \\
30(49.2) \\
2(3.3)\end{array}$ & $\begin{array}{c}50(70) \\
34(47.2) \\
31(43) \\
23(32)\end{array}$ \\
\hline $\begin{array}{l}\text { Localización del dolor } \ddagger \text {, }(\%) \\
\text { Retroesternal } \\
\text { Tórax anterior } \\
\text { Hombro } \\
\text { Cuello } \\
\text { Epigastrio }\end{array}$ & $\begin{array}{c}108(81.2) \\
13(9.8) \\
2(1.5) \\
3(2.3) \\
10(7.5)\end{array}$ & $\begin{array}{l}55(90.2) \\
5(8.2) \\
1(1.6) \\
1(1.6) \\
3(4.9)\end{array}$ & $\begin{array}{l}53(73.6) \\
8(11.1) \\
1(1.4) \\
2(2.8) \\
7(9.7)\end{array}$ \\
\hline $\begin{array}{l}\text { Irradiación del dolor } ¥, \mathrm{n}(\%) \\
\text { Cuello } \\
\text { Mandíbula } \\
\text { Hombro derecho } \\
\text { Hombro izquierdo } \\
\text { Brazo derecho } \\
\text { Brazo izquierdo } \\
\text { Dorso } \\
\text { Otro } \\
\text { No refiere }\end{array}$ & $\begin{aligned} 20 & (15) \\
17 & (12.9) \\
3 & (2.3) \\
13 & (9.8) \\
7 & (5.2) \\
36 & (27.1) \\
20 & (15.1) \\
7 & (5.2) \\
48 & (36.1)\end{aligned}$ & $\begin{array}{c}6(9.8) \\
7(11.5) \\
1(1.6) \\
7(11.5) \\
5(8.2) \\
20(32.8) \\
9(14.7) \\
3(4.9) \\
19(31.1)\end{array}$ & $\begin{aligned} & 14(19.4) \\
& 10(13.9) \\
& 2(2.7) \\
& 6(8.3) \\
& 2(2.8) \\
& 16(22.2) \\
& 11(15.3) \\
& 4(5.5) \\
& 29(40.3)\end{aligned}$ \\
\hline $\begin{array}{l}\text { Intensidad del dolor torácico, } \\
\text { promedio (DE) }\end{array}$ & $7.8(2.1)$ & $8.4(1.7)$ & $7.1(1.9)$ \\
\hline $\begin{array}{l}\text { Antecedentes personales } \S, \mathrm{n}(\%) \\
\text { Hipertensión arterial } \\
\text { Diabetes mellitus tipo } 2 \\
\text { Insuficiencia cardiaca } \\
\text { Accidente cerebrovascular } \\
\text { Enfermedad arterial oclusiva periférica } \\
\text { Dislipidemia } \\
\text { Fibrilación auricular } \\
\text { Enfermedad coronaria } \\
\text { Antecedente familiar enfermedad } \\
\text { coronaria } 9 \\
\text { Uso de ASA } \\
\text { Consumo de cocaína } \\
\text { Revascularización miocárdica ** } \\
\text { Tabaquismo } \\
\text { Diálisis } \\
\text { Antecedente de angioplastia } \\
\text { Enfermedad renal }\end{array}$ & $\begin{array}{c}84(63.2) \\
20(15.1) \\
6(4.5) \\
7(5.2) \\
0 \\
48(36.1) \\
4(3) \\
38(28.5) \\
18(13.5) \\
37(27.9) \\
1(0.7) \\
13(9.8) \\
66(49.6) \\
2(1.5) \\
15(11.2) \\
7(5.2)\end{array}$ & $\begin{array}{c}32(52.4) \\
10(16.4) \\
1(1.6) \\
4(6.6) \\
0 \\
15(24.6) \\
2(3.2) \\
15(24.6) \\
8(13.1) \\
13(21.3) \\
1(1.6) \\
4(6.6) \\
30(49.2) \\
1(1.6) \\
6(9.8) \\
1(1.6)\end{array}$ & $\begin{array}{c}52(72.2) \\
10(13.9) \\
5(7) \\
3(4.2) \\
0 \\
33(45.8) \\
2(2.8) \\
23(32) \\
10(14) \\
\\
24(33.3) \\
0 \\
9(12.5) \\
36(50) \\
1(1.4) \\
9(12.5) \\
6(8.3)\end{array}$ \\
\hline \multicolumn{4}{|c|}{$\begin{array}{l}\text { *Un paciente refirió dolor torácico opresivo y urente. Cinco pacientes negaban presencia } \\
\text { de dolor torácico } \\
\text { †Asociación del dolor torácico con la presencia de síntomas disautonómicos. } \\
\text { † Un paciente podía presentar diferentes sitios de localización y de irradiación del dolor } \\
\text { J Muerte por enfermedad coronaria en familiar de primer grado: mujeres menores de } \\
65 \text { años, hombres menores de } 55 \text { años. } \\
\text { ** Percutánea o quirúrgica }\end{array}$} \\
\hline
\end{tabular}


de $90 \%)$ en $18 \%(n=22)$ (Tabla 2). La distribución de los casos de IMEST de acuerdo con la escala de estratificación de riesgo de Killip KimbalI, II, III y IV, fue 72.1, 6.6, 16.4 y $4.9 \%$ respectivamente.

\section{Hallazgos en el EKG}

En el 48.1\% (n=64) de los casos se observó en el EKG de ingreso elevación del segmento ST, inversión de la onda $\mathrm{T}$ en $20.3 \%(\mathrm{n}=27)$, descenso del segmento ST en $14.2 \%$ $(n=19)$ y fue considerado normal en $17.3 \%(n=23)$. Dentro de los IMEST la localización más frecuente fue la pared inferior $50.8 \%(\mathrm{n}=31)$ seguido por la anterior $34.4 \%(\mathrm{n}=21)$ y en $8.1 \%(n=5)$ se consideró un infarto extenso.

Tabla 2. Hallazgos clínicos y paraclínicos al ingreso de los pacientes con síndrome coronario agudo.

\begin{tabular}{|c|c|c|c|}
\hline & $\begin{array}{c}\text { TOTAL } \\
133\end{array}$ & $\begin{array}{c}\text { IMEST } \\
61\end{array}$ & $\begin{array}{c}\text { SCASEST } \\
72\end{array}$ \\
\hline $\begin{array}{l}\text { Presión arterial sistólica, n (\%) } \\
\quad<90 \\
90-139 \\
>140\end{array}$ & $\begin{array}{c}7(5.3) \\
73(55.3) \\
52(39.3)\end{array}$ & $\begin{array}{c}0 \\
35(53.7) \\
26(42.6)\end{array}$ & $\begin{array}{c}7(9.7) \\
38(52.7) \\
26(36.1)\end{array}$ \\
\hline $\begin{array}{l}\text { Presión arterial diastólica, n (\%) } \\
\quad<60 \\
\quad 61-100 \\
>100\end{array}$ & $\begin{array}{c}10(7.6) \\
109(82.6) \\
13(10)\end{array}$ & $\begin{array}{c}1(1.6) \\
54(88.5) \\
6(9.8)\end{array}$ & $\begin{array}{l}9(12.5) \\
55(76.3) \\
7(9.7)\end{array}$ \\
\hline $\begin{array}{l}\text { Frecuencia cardíaca, n (\%) } \\
\quad<60 \\
60-100 \\
>100\end{array}$ & $\begin{array}{l}28(21.2) \\
90(68.2) \\
14(10.6)\end{array}$ & $\begin{array}{c}10(16.3) \\
45(73.7) \\
6(9.8)\end{array}$ & $\begin{array}{c}18(25) \\
45(22.5) \\
8(11.1)\end{array}$ \\
\hline Saturación de oxígeno $<90 \%, \mathrm{n}(\%)$ & $22(18)$ & $6(9.8)$ & $16(22.2)$ \\
\hline $\begin{array}{l}\text { Hallazgos a la auscultación cardiaca n (\%) } \\
\text { Presencia de estertores, n (\%) } \\
\text { Tercer ruido cardiaco } \\
\text { Soplo mitral }\end{array}$ & $\begin{array}{c}20(15.1) \\
2(1.5) \\
4(3)\end{array}$ & $\begin{array}{c}7(11.1) \\
0 \\
2(3.2)\end{array}$ & $\begin{array}{l}13(18) \\
2(2.7) \\
2(2.7)\end{array}$ \\
\hline $\begin{array}{l}\text { Hallazgos en EKG de ingreso, n (\%) } \\
\text { Rectificación del ST } \\
\text { Elevación del ST } \\
\text { Extensión a ventrículo derecho } \\
\text { Onda T picuda } \\
\text { Descenso del ST } \\
\text { Inversión de la onda T }\end{array}$ & $\begin{array}{c}15(11.2) \\
64(48.1) \\
6(4.5) \\
12(9) \\
19(14.2) \\
27(20.3)\end{array}$ & $\begin{array}{c}2(3.2) \\
61(100) \\
6(9.8) \\
4(6.5) \\
2(3.2) \\
7(11.1)\end{array}$ & $\begin{array}{c}13(18) \\
0 \\
0 \\
8(11.1) \\
17(23.6) \\
20(27.7)\end{array}$ \\
\hline $\begin{array}{l}\text { Pared comprometida } \S \S, \mathrm{n}(\%) \\
\text { Pared inferior } \\
\text { Pared lateral } \\
\text { Pared anterior } \\
\text { Pared septal } \\
\text { Pared posterior } \\
\text { Extenso }\end{array}$ & $\begin{array}{l}- \\
- \\
- \\
- \\
-\end{array}$ & $\begin{array}{l}31(50.8) \\
14(23) \\
21(34.4) \\
17(37.8) \\
4(6.5) \\
5(8.1)\end{array}$ & $\begin{array}{l}- \\
- \\
- \\
- \\
-\end{array}$ \\
\hline $\begin{array}{l}\text { Hallazgos en el ecocardiograma TTIJ, n (\%) } \\
\text { Trastorno de contractilidad } \\
\text { Cardiopatía isquémica } \\
\text { Cardiopatía dilatada } \\
\text { Cardiopatía hipertensiva } \\
\text { Valvulopatía mitral } \\
\text { Normal } \\
\text { No realizado }\end{array}$ & $\begin{array}{l}52(39.1) \\
67(50.3) \\
3(2.2) \\
39(29.3) \\
19(14.2) \\
6(4.51) \\
42(31.5)\end{array}$ & $\begin{array}{c}29(47.5) \\
38(62.2) \\
2(3.2) \\
16(26.2) \\
11(18) \\
0 \\
21(34.4)\end{array}$ & $\begin{array}{c}23(32) \\
29(40.2) \\
1(1.3) \\
23(31.9) \\
8(11.1) \\
6(8.3) \\
21(29.1)\end{array}$ \\
\hline \multicolumn{4}{|c|}{$\begin{array}{l}\text { I Troponina T, medida con método de ELISA, positiva nivel mayor de } 0.4 \mathrm{ng} / \mathrm{mL} \text {. } \\
\text { Se obtuvieron datos incompletos ya que durante } 2009 \text { la troponina no se encontraba } \\
\text { incluida en el POS } \\
\text { §§Valorada con electrocardiograma de ingreso } \\
\text { IJ Algunos pacientes tienen más de una alteración identificada en el ecocardiograma } \\
\text { transtorácico }\end{array}$} \\
\hline
\end{tabular}

\section{Tratamiento farmacológico}

En cuanto al manejo médico de ingreso, $88 \%(\mathrm{n}=117)$ recibieron inhibidores de la enzima convertidora de angiotensina (IECA), 5.2\% $(\mathrm{n}=7)$ antagonistas de los receptores de angiotensina II (ARAII), 93.2\% $(\mathrm{n}=124)$ betabloqueadores (BB), 97.7\% ( $\mathrm{n}=130)$ ASA, 87.2\% $(\mathrm{n}=116)$ clopidogrel y $92.4 \%(n=123)$ estatinas. Se ordenó heparina de bajo peso molecular (enoxaparina en todos los casos) en $94.7 \%$ $(\mathrm{n}=126)$ con bolo inicial en sólo $3.7 \%(\mathrm{n}=5)$ (Tabla 3).

\section{Terapia de reperfusión y procedimientos invasivos}

Entre los pacientes con diagnóstico de IMEST cuyos síntomas se encontraban en ventana para trombolísis y no presentaban contraindicaciones, se trombolizaron $82.6 \%$ $(n=38$ de 46). En un paciente se practicó angioplastia primaria como estrategia de reperfusión. Se realizó cateterismo cardiaco en $79.6 \%$ de los casos $(n=82$ de 103 pacientes que no fueron remitidos). La principal arteria comprometida fue la coronaria derecha $47.5 \%(n=39)$, seguida de la descendente anterior $40.2 \%(n=33)$; hubo compromiso de dos vasos en $11 \%(\mathrm{n}=9)$ y de tres vasos $4.9 \%(\mathrm{n}=4)$, finalmente se observó en un $4.9 \%(n=4)$ de los pacientes coronarias sanas. Se practicó angioplastia en 49 pacientes, de los cuales 12 se realizaron antes de 72 horas y 37 después de las 72 horas. Fueron llevados a cirugía de derivación coronaria $3.8 \%(\mathrm{n}=4)$ (Tabla 3$)$.

Tabla 3. Tratamiento e intervenciones realizadas.

\begin{tabular}{|c|c|c|c|}
\hline & $\begin{array}{c}\text { TOTAL } \\
133\end{array}$ & $\begin{array}{c}\text { IMEST } \\
61\end{array}$ & $\begin{array}{c}\text { SCASEST } \\
72\end{array}$ \\
\hline $\begin{array}{l}\text { Manejo farmacológico de ingreso, } \mathrm{n} \text { (\%) } \\
\text { Oxígeno } \\
\text { IECA } \\
\text { ARA II } \\
\text { Betabloqueador } \\
\text { Estatina } \\
\text { Nitrato } \\
\text { Morfina } \\
\text { ASA } \\
\text { Clopidogrel } \\
\text { Heparina de bajo peso molecular } \\
\text { Inhibidores glico-proteínas IIB/IIIA } \\
\text { Heparina en bolo }\end{array}$ & $\begin{array}{c}133(100) \\
117(88) \\
7(5.2) \\
124(93.2) \\
123(92.4) \\
64(48.1) \\
53(40) \\
130(97.7) \\
116(87.2) \\
126(94.7) \\
0 \\
5(3.7)\end{array}$ & $\begin{array}{c}61(100) \\
51(83.6) \\
6(10) \\
57(93.4) \\
55(90.1) \\
38(62.2) \\
25(41) \\
59(96.7) \\
60(98.3) \\
60(98.3) \\
0 \\
4(6.5)\end{array}$ & $\begin{array}{c}72(100) \\
66(91.6) \\
1(1.3) \\
67(93) \\
68(94.4) \\
26(36.1) \\
25(34.7) \\
71(98.6) \\
54(75) \\
66(91.6) \\
0 \\
1(1.3)\end{array}$ \\
\hline Realización de trombolisis $\dagger$ & $38(28.5)$ & $38(82.6)$ & \\
\hline $\begin{array}{l}\text { Angioplastia } \neq \\
\text { Tiempo de angioplastia }>72 \text { horas } \\
\text { Tiempo de angioplastia }<72 \text { horas }\end{array}$ & $\begin{array}{c}57(42.8) \\
12(9)\end{array}$ & $\begin{array}{l}33(54) \\
6(9.8)\end{array}$ & $\begin{array}{c}24(33.3) \\
5(6.9)\end{array}$ \\
\hline $\begin{array}{l}\text { Coronaria comprometida } \\
\text { coronaria derecha } \\
\text { Descendente anterior } \\
\text { Circunfleja } \\
\text { Otra } \\
\text { Coronarias sanas }\end{array}$ & $\begin{array}{c}39(47.5) \\
33(40.2) \\
20(24.3) \\
19(23.1) \\
4(4.8)\end{array}$ & $\begin{array}{l}24(54.5) \\
18(41) \\
8(18.1) \\
10(22.7) \\
1(2.2)\end{array}$ & $\begin{array}{l}15(40.5) \\
15(40.5) \\
12(50) \\
9(24.3) \\
2(5.4)\end{array}$ \\
\hline \multicolumn{4}{|c|}{$\begin{array}{l}\text { *Evidenciado en ecocardiograma transtorácico. } \\
\dagger \text { Pacientes que cumplían criterios: menos de } 12 \text { horas de evolución, sin contraindica- } \\
\text { ciones absolutas ni relativas. } \\
\ddagger \text { Se tienen en cuenta las angioplastias realizadas en el HSJ y no las realizadas en otra } \\
\text { institución. }\end{array}$} \\
\hline
\end{tabular}




\section{Eventos cardiovasculares posteriores y evolución hospitalaria}

$\mathrm{Al}$ hacer el análisis de los desenlaces cardiovasculares mayores, se obtuvo: muerte en $8.7 \%(\mathrm{n}=9)$ de los pacientes, nuevo infarto $8.7 \%(n=9)$, choque cardiogénico $8.7 \%(n=9)$, revascularización de vaso previo $0.97 \%(\mathrm{n}=1)$ (Tabla 4). La mayoría de los pacientes fueron trasladados a UCI 90.2\% $(n=93)$, y 30 pacientes fueron remitidos debido a la falta de disponibilidad de camas dentro de la institución, por lo que no se tuvieron en cuenta para la definición de los desenlaces.

\section{Discusión}

En los resultados encontramos una mayor prevalencia de angina inestable/IMSEST con respecto al IMEST. Estos datos coinciden con la mayoría de registros en los cuales la prevalencia del SCA sin elevación del segmento ST (SCA SEST) es mayor. En The Global Registry of Acute Coronary Events (GRACE) se encontró en 63\% (9), en el registro GRACE expandido 58\% (10) y en el ACCESS 54\% (11). Por su parte, el registro mexicano RENASICA II mostró mayor número de pacientes con SCA con elevación del ST con $56.2 \%$ (12). Si bien es cierto que los porcentajes varían en un rango amplio, es importante anotar que la forma de recolección de la información y los criterios diagnósticos utilizados influyen en estas proporciones. En nuestro estudio se pudo generar un subregistro en los pacientes con angina inestable, considerando que algunos de éstos quedan con diagnóstico de dolor torácico y pueden haber sido estudiados de forma ambulatoria.

La mayoría de los pacientes consultó por episodio único de dolor torácico típico dentro de las primeras seis horas del inicio del cuadro clínico, clínicamente estables al ingreso, mientras que pocos pacientes no refirieron el dolor. Las diversas revisiones sistemáticas acerca del comportamiento clínico de los SCA apuntan a que no es posible definir un rol importante para un único síntoma o signo clínico en el diagnóstico de los SCA (13-16), sus reportes y otros (17-19) insisten en la importancia del enfoque clínico en la orientación diagnóstica inicial, por lo que sigue siendo importante la información concerniente al comportamiento clínico de ellas.

Observamos que muchos pacientes con diagnóstico inicial de SCA terminaron con un diagnóstico de egreso diferente de estas patologías y tres pacientes presentaron lecturas erróneas de elevación del segmento ST en el EKG de ingreso, lo cual resalta la dificultad en llegar al diagnóstico definitivo en algunos casos. Esto ha sido visto en registros como el GRACE, en el cual $42 \%$ de los pacientes con diagnóstico inicial de angina inestable terminaron con diagnósticos de egreso diferentes de SCA (9). En el registro europeo (Euro Heart) se observó una situación semejante (20).

En cuanto al tratamiento farmacológico, se encontró una adecuada adherencia a las recomendaciones de las guías. La formulación de aspirina, betabloqueadores, IECA o ARAII y anticoagulantes fue mayor a $90 \%$, porcentaje semejante a
Tabla 4. Desenlaces cardiovasculares mayores (MACE) durante la hospitalización en pacientes que no fueron remitidos.

\begin{tabular}{|l|c|c|c|}
\hline & $\begin{array}{c}\text { TOTAL } \\
\mathbf{1 0 3}\end{array}$ & $\begin{array}{c}\text { IMEST } \\
\mathbf{4 5}\end{array}$ & $\begin{array}{c}\text { SCASEST } \\
\mathbf{5 8}\end{array}$ \\
\hline Descelace MACE, $\mathbf{n}(\%)$ & & & \\
Muerte & $9(8.7)$ & $3(6.7)$ & $6(10.3)$ \\
Nuevo infarto & $9(8.7)$ & $7(15.6)$ & $5(8.6)$ \\
Revascularización vaso previo & $1(0.9)$ & $1(2.2)$ & 0 \\
Cirugía & $4(3.8)$ & $1(2.2)$ & $3(5.2)$ \\
Choque cardiogénico & $9(8.7)$ & $3(6.7)$ & $6(10.3)$ \\
\hline Destino del paciente, n (\%) & & & \\
Traslado a UCI (\% sobre el total 133) & $93(69.9)$ & $45(73.7)$ & $48(66.6)$ \\
Remitido (\% sobre el total 133) & $30(22.6)$ & $16(26.2)$ & $14(19.4)$ \\
\hline
\end{tabular}

lo reportado en registros internacionales como el GRACE expandido (10), el Euro Heart 2 (21) y The National Registry of Myocardial Infarction (NRMI 1-5) (22). Se utilizó HBPM en un porcentaje alto comparado con 41-51\% en el GRACE (9), 47.8-58.1\% en el Euro Heart (20) y 61.1-61.4\% en el ACCESS (11). Esto puede estar relacionado con la mayor facilidad para su administración, la evidencia creciente de su eficacia y seguridad (3-5) y el fácil acceso a este recurso en la institución.

El uso de clopidogrel estuvo en un porcentaje alto comparado con otros registros, sin embargo la evidencia del beneficio clínico de este medicamento en todo el espectro del SCA y la adopción de ellos por parte de guías de manejo es reciente $(23,24)$. Por lo tanto, su uso ha aumentado progresivamente en los últimos años, lo cual se evidencia en registros como GRACE expandido y NRMI 1-5 $(10,25)$. Los resultados comparativos con otros registros acerca de los manejos y desenlaces se encuentran resumidos en la Tabla 5 .

Con respecto a la reperfusión, se practicó fibrinólisis en $82.6 \%$ de los pacientes que tenían indicación. Este porcentaje es alto comparado con otras experiencias en Latinoamérica como el estudio RENASICA II en donde se trombolizó $37 \%$ de los pacientes con IMEST y en un 15\% adicional se practicó angioplastia primaria o facilitada (12). En el registro peruano de infarto de miocardio (RENIMA) se realizó trombolísis a $14.1 \%$ de los casos y angioplastia en $11.9 \%$. Aunque no se especifica el porcentaje de pacientes con IMEST que se trombolizó, esta población representó $53.1 \%$ del registro (25). Comparado con registros internacionales, el porcentaje de trombolísis en nuestro estudio fue superior. En el registro Euro Heart 2, 63.9\% recibieron un tratamiento de reperfusión primaria (21), mientras que en el GRACE, 33\% de los pacientes no recibieron ninguna estrategia de reperfusión (6).

Es importante mencionar que en un sólo paciente se practicó angioplastia primaria como estrategia de reperfusión. $\mathrm{Al}$ respecto, nuestra institución cuenta con laboratorio de cateterismo cardiaco; sin embargo, su disponibilidad no es de 24 horas, por lo que se encuentra limitada la posibilidad de realizar angioplastia primaria. Sin embargo, experiencias internacionales muestran cómo a lo largo de los años la utilización de la angioplastia primaria ha aumentado progre- 
Tabla 5. Tabla comparativa de principales registros en pacientes con síndrome coronario agudo.

\begin{tabular}{|c|c|c|c|c|c|c|}
\hline & & HSJ & $\begin{array}{c}\text { ACCES } \\
\text { (COLOMBIA) }\end{array}$ & EURO HEART2 & NRMI 5 (USA) & ACCES \\
\hline & & 2013,133 ptes & 2012,790 ptes & 2006,6385 ptes & 2008,347286 ptes & 2011,11731 ptes \\
\hline Aspirina & $\begin{array}{l}\text { IMEST } \\
\text { AI/IMSEST }\end{array}$ & $\begin{array}{l}96 \\
98\end{array}$ & 95 & $\begin{array}{l}96.8 \\
94.5\end{array}$ & $\begin{array}{c}90 \\
-\end{array}$ & $\begin{array}{l}94 \\
92\end{array}$ \\
\hline Betabloqueadores & $\begin{array}{l}\text { IMEST } \\
\text { AI/IMSEST }\end{array}$ & $\begin{array}{c}93.4 \\
95\end{array}$ & 92.8 & $\begin{array}{c}83 \\
82.8\end{array}$ & $\begin{array}{c}82 \\
-\end{array}$ & $\begin{array}{l}78 \\
78\end{array}$ \\
\hline IECA/BRA II & $\begin{array}{l}\text { IMEST } \\
\text { AI/IMSEST }\end{array}$ & $\begin{array}{l}83.6 \\
94.2\end{array}$ & 79 & $\begin{array}{l}75.4 \\
69.5\end{array}$ & $\begin{array}{l}74 \\
-\end{array}$ & $\begin{array}{c}82.2 \\
76\end{array}$ \\
\hline Clopidogrel (o ticlopidina) & $\begin{array}{l}\text { IMEST } \\
\text { AI/IMSEST }\end{array}$ & $\begin{array}{l}98.3 \\
86.5\end{array}$ & 79.5 & $\begin{array}{l}69.8 \\
67.4\end{array}$ & $\begin{array}{l}- \\
-\end{array}$ & $\begin{array}{l}84 \\
78\end{array}$ \\
\hline Anticoagulación & $\begin{array}{l}\text { IMEST } \\
\text { AI/IMSEST }\end{array}$ & $\begin{array}{l}98 \\
96\end{array}$ & 97.2 & $\begin{array}{l}77.2 \\
72.9\end{array}$ & $\begin{array}{c}80 \\
-\end{array}$ & $\begin{array}{l}- \\
-\end{array}$ \\
\hline Estatina & $\begin{array}{l}\text { IMEST } \\
\text { AI/IMSEST }\end{array}$ & $\begin{array}{c}90.1 \\
98\end{array}$ & 94.6 & $\begin{array}{l}80.7 \\
73.7\end{array}$ & $\begin{array}{c}88 \\
-\end{array}$ & $\begin{array}{l}91 \\
90\end{array}$ \\
\hline Angiografía & $\begin{array}{l}\text { IMEST } \\
\text { AI/IMSEST }\end{array}$ & 79.6 & 83.1 & $\begin{array}{l}70.2 \\
62.9\end{array}$ & $\begin{array}{c}86 \\
-\end{array}$ & $\begin{array}{l}56 \\
59\end{array}$ \\
\hline Angioplastia & $\begin{array}{l}\text { IMEST } \\
\text { AI/IMSEST }\end{array}$ & $\begin{array}{l}39 \\
36\end{array}$ & 46.5 & $\begin{array}{l}57.8 \\
37.1\end{array}$ & $\begin{array}{l}73 \\
-\end{array}$ & $\begin{array}{l}40 \\
31\end{array}$ \\
\hline CRVM & $\begin{array}{l}\text { IMEST } \\
\text { AI/IMSEST }\end{array}$ & $\begin{array}{l}1.6 \\
4.1\end{array}$ & 12.2 & $\begin{array}{l}2.9 \\
7.4\end{array}$ & $\begin{array}{l}7 \\
-\end{array}$ & $\begin{array}{l}3.8 \\
7.3\end{array}$ \\
\hline Trombolisis* & & 82.6 & - & 26.3 & - & 39 \\
\hline PCI primaria* & & 0.97 & - & 37.6 & - & 35 \\
\hline Trombolisis o angioplastia* & & 83.3 & - & 63.9 & - & 61 \\
\hline Mortalidad global & & 8.7 & 8.3 & 4 & 6.3 & 3.6 \\
\hline
\end{tabular}

sivamente como estrategia de reperfusión en pacientes con IMEST (21). Por lo tanto, en nuestro hospital se debe insistir en este recurso ya que en muchos casos es la estrategia de reperfusión de elección para el manejo de estos pacientes.

En Colombia, desafortunadamente no se cuenta con un registro nacional de SCA que nos proporcione datos acerca de la atención de estos pacientes, aunque a nivel mundial la importancia de los registros está bien establecida $(7,26)$. Sin embargo hospitales de nuestro país han participado en registros internacionales que han permitido obtener datos para nuestra población (27) y adicionalmente se conocen reportes de estudios importantes que han dado información acerca del tratamiento y la mortalidad en estos pacientes $(28,29)$.

El presente estudio tiene una fortaleza importante en los datos clínicos que se reportan y son escasos en otros registros evaluados. Por otra parte, el estudio presenta algunas debilidades. La principal es su carácter retrospectivo que limita la adquisición de información importante en cuanto a datos clínicos y ciertos aspectos terapéuticos como el uso de fármacos en función de sus contraindicaciones, el tiempo puerta aguja, tipo de trombolítico utilizado y uso de stent. Adicionalmente puede existir un posible sesgo de selección en cuanto a la identificación de los pacientes ya que puede haber pacientes con SCA atendidos no identificados con los códigos CIE 10 utilizados para tal efecto. Tampoco fue posible analizar posibles contraindicaciones en la utilización de medicamentos ordenados en este contexto. Finalmente el estudio se realizó en una sola institución universitaria de tercer nivel, lo cual limita la validez externa de estos resultados.

\section{Conclusión}

En la población estudiada predominó el síndrome coronario agudo sin elevación del segmento ST. La mayoría de pacientes consultaron por dolor torácico retroesternal, en las primeras horas de inicio. El porcentaje de uso de gran parte de los medicamentos para el tratamiento de estas patologías fue semejante al reportado en registros importantes a nivel mundial. El porcentaje de reperfusión en pacientes con infarto con supradesnivel del segmento ST fue alto, con uso 
predominante de trombolisis farmacológica; sin embargo, aun tenemos pacientes que no recibieron el beneficio de esta terapia y el uso de angioplastias primarias fue muy bajo.

\section{Conflicto de interés}

Los autores del presente trabajo no presentan ningún conflicto de interés.

\section{Referencias}

1. Global Health Observatory Data Repository. Mortality: Cardiovascular diseases and diabetes, deaths per 100.000 www.who.int/gho/ncd/mortality/cvd/ en/index.html2013.

2. DANE. Defunciones por grupos de edad y sexo según departamento, municipio y residencia y grupos de causa de defunción 2009-2010. www.dane.gov.co [cited 2012].

3. Beltrán J B, Caicedo V, Garcìa M, García E, et al. Guías colombianas de cardiología. Síndrome coronario agudo sin elevación del ST . Rev Col Card 2008.

4. Scott R AJ, Bachelder B, Adams C. et al. Focused update of the guidelines for the management of patients with unstable angina/Non-ST elevation myocardial infarction. J Am Coll Cardiol 2011; 57 (19): 1920-59.

5. Steg G JS, Atar D, Badano L, Blomstrom BM, et al. Guidelines for the management of acute myocardial infarction in patients presenting with ST-segment elevation. Eur Heart J 2012; 33 (20): 2569-619.

6. Eagle K NB, Mehta R, Granger C, Steg P, Van de werf F, et al. Trends in acute reperfusion therapy for ST-segment elevation myocardial infarction from 1999 to 2006: we are getting better but we have got a long way to go. Eur Heart $J$ 2008; (29): 609-17.

7. Zeymer U SJ. Why do we need prospective registries in patients with acute myocardial infarction? Eur Heart J 2003; 24: 1611-2.

8. Erhardt L. HJ, Bossaert L., Halinen M., Keltai M., Koster R., Marcassa C., Quinn T. y van Weert H. European Society of Cardiology: Task force on the management of chest pain. Eur Heart J 2002; 23: 1153-76.

9. Steg P GR, Gore j, Fox K, Eagle K, Flather M, et al. Baseline Characteristics, Management, Practices, and In-Hospital Outcomes of Patients Hospitalized with Acute Coronary syndromes in the Global Registry of Acute Coronary Events (GRACE). Am J Cardiol 2002; 90: 358-63.

10. Goodman S HW, Yan A, Budaj A, Kennelly B, Gore J, et al. The expanded Global Registry of acute Coronary Events: Baseline characteristics, management practices, and hospital outcomes of patients with acute coronary syndromes. Am Heart J 2009; 158: 193-201.

11. Investigators TA. Management of acute coronary syndromes in developing countries: Acute Coronary Events - a multinational Survey of current management Strategies. Am Heart J 2011; 162: 852-9.

12. García-Castillo A, Martínez Bermúdez P, et al. RENASICA II (Registro Mexicano de Síndromes Coronarios). Archivos de Cardiología de México 2001; 75 (1).

13. Panju A HB, Guyatt G, Simel D. The rational clinical examination. Is this patient having a myocardial infarction? . JAMA 1998; 280 (14): 1256-63.
14. Goodacre S LT, Morris F, Campbell S. How useful are clinical features in the diagnosis of acute,undifferentiated chest pain?. Acad Emerg Med 2002; 9: 203-8.

15. Swap CJ NJ. Value and limitations of chest pain history in the evaluation of patients with suspected acute coronary syndromes. JAMA 2005; 294 (20): 2623-9.

16. Bruyninckx R AB, Bruyninckx P, Buntinx F. Signs and symptoms in diagnosing acute myocardial infarction and acute coronary syndrome: a diagnostic meta-analysis. Br J Gen Pract 2008; 58 (547): 105-11.

17. Lee TH, Weisberg MC, et al. Clinical characteristics and natural history of patients with acute myocardial infarction sent home from the emergency room. Am J Cardiol 1987; 60: 219-24.

18. Solomon CG LT, Cook EF, et al. Comparison of clinical presentation of acute myocardial infarction in patients older than 65 years of age to younger patients: the Multicenter Chest Pain Study experience. Am J Cardiol 1989; 63: 772-6.

19. Everts B KB, Wahrborg P, et al. Localization of pain in suspected acute myocardial infarction in relation to final diagnosis, age and sex, and site and type of infarction. Heart Lung 1996; 25: 430-7.

20. Hasdai D, Wallentin L, Danchin N, Gitt AK, Boersma E, et al. A prospective survey of the characteristics, treatments and outcomes of patients with acute coronary syndromes in Europe and the Mediterranean basin. Eur Heart 2002; 23: $1190-201$

21. Mandelzweig L BA, Boyko V, Bueno H, Danchin N, Filippatos G, et al. The second Euro Heart Survey on acute coronary syndromes: characteristics, treatment, and outcome of patients with ACS in Europe and the Mediterranean Basin in 2004. Eur Heart J 2006; 27: 2285-93.

22. Peterson E SB, Parson L, Pollack Ch, French W, Canto J, et al. Rends in quality of care for patients with acute myocardial infarction in the National Registry of Myocardial Infarction from 1990 to 2006. Am Heart J. 2008;156:1045-55.

23. Chen ZM, Jiang LX, Chen YP, Xie JX, Pan HC, Peto R, et al. COMMIT (ClOpidogrel and Metoprolol in Myocardial Infarction Trial) collaborative group. Addition of clopidogrel to aspirin in 45852 patients with acute myocardial infarction: randomised, placebo-controlled trial. Lancet. 2005; 366: 1607-21.

24. Sabatine MS CC, Gibson CM, et al. Addition of clopidogrel to aspirin and fibrinolytic therapy for myocardial infarction with ST-segment elevation. NEngl J Med 2005; 352: 1179-89.

25. Miguel Reyes Rocha JHL, Santiago Campodónico Hoyos, José Drago Silva y Oscar Alvarado Contreras. Registro Nacional de Infarto Miocárdico Agudo (RENIMA). Revista Peruana de Cardiología 2008: 85-99.

26. Fox KA, Cokkinos DV, Deckers J, Keil U, Maggioni A, Steg G. On belhaf of the ENACT (European Network for acute coronary treatment investigators) The ENACT study: a pan-European survey of acute coronary syndromes. Eur Heart 2000; 21: 1440-9.

27. Isaza VD, Plata E. ACCES: En cuesta de estrategia de manejo en síndrome coronario agudo (SCA). Resultados de los 12 meses de seguimiento en la población de Colombia comparada con Latinoamérica. Rev Col Cardiol 2012: 35

28. Casas JP C-GL, Villa-Roel C, et al. Quality of care and cardiovascular events in AMI patients from a developing country. Circulation 2002; (106): e123.

29. Chavarriaga J BJ, Senior JM. Características epidemiológicas, clínicas, tratamiento y pronóstico de los pacientes con síndrome coronario agudo (SCA) en la Fundación Hospitalaria San Vicente de Paul (FHSVP). Rev Col Cardiol 2012; 19 (supl 1): 38 . 